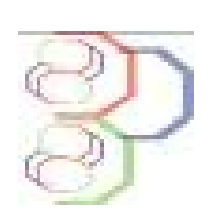

Journal of Applied Biosciences 126: 12638-12647

\title{
Perception paysanne des principales contraintes et pratiques culturales en production de patate douce au Burkina Faso.
}

\author{
1,2Souleymane KOUSSOUBE, ${ }^{1 *}$ Fousséni TRAORE, ${ }^{1}$ Koussao SOME, ${ }^{1}$ Clémentine BINSO-DABIRE, \\ ${ }^{2}$ Antoine SANON. \\ 1'Institut de l'Environnement et de Recherches Agricoles, CREAF de Kamboinsé, 01 BP 476 Ouagadougou 01, \\ Burkina Faso ; \\ ${ }^{2}$ Laboratoire d'Entomologie Fondamentale et Appliquée, Unité de Formation et de Recherches en Sciences de la Vie \\ et de la Terre, Université Ouaga I Pr Joseph Ki-Zerbo, 06 BP 9499 Ouagadougou 06, Burkina Faso. \\ *Auteur correspondant Tel : + 22676659422 ; Email : foussnitraore@gmail.com
}

Original submitted in on 14th March 2018. Published online at www.m.elewa.org on $30^{\text {th }}$ June 2018 https://dx.doi.org/10.4314/jab.v126i1.2

\section{RÉSUMÉ}

Objectif : la production de la patate douce, Ipomoea batatas (L.) Lam., reste confrontée à de nombreuses contraintes surtout d'ordre biotique. Pour mieux faire face à ces contraintes, il est indispensable de les connaitre avec précision et de les hiérarchiser.

Méthodologie et résultats : une enquête a été réalisée entre juillet 2012 et octobre 2016, auprès de 200 producteurs dans les cinq principales provinces de production de la patate douce au Burkina Faso (Gourma, Kénédougou, Kouritenga, Nahouri, Sissili). Les résultats indiquent que pour les producteurs, les insectes constituent la contrainte biotique majeure avec des perceptions variables d'une province à l'autre. Ainsi, le charançon Cylas puncticollis est perçu comme le premier ravageur des feuilles dans le Nahouri $(52,94 \%)$, le Kouritenga et le Gourma $(75,51 \%)$ alors que dans la Sissili et le Kénédougou, c'est plutôt l'espèce de mouche blanche, Bemisia tabaci qui occupe cette position. Cependant, le charançon est perçu comme le ravageur le plus important des tubercules dans toutes les provinces. II est aussi considéré par au moins $50 \%$ des répondants comme le principal ravageur des tiges. Les pratiques culturales sont caractérisées par une forte application de fumure organique, de NPK et d'urée en fonction des zones. La rotation des cultures et l'utilisation des insecticides chimiques sont citées comme méthodes de contrôle des principaux ennemis de la patate douce.

Conclusion et application des résultats : cette étude montre que les producteurs ont une bonne connaissance des contraintes biotiques liées à la production de la patate. Ce qui est de nature à faciliter la mise au point de stratégies participatives et efficaces de gestion durable du charançon au Burkina Faso. Les résultats alimenteront les réseaux d'encadrement des producteurs et serviront à mieux définir la stratégie nationale de développement de la filière.

Mots clés : Burkina Faso, patate douce, contraintes de production, insectes ravageurs, Cylas puncticollis, perception des producteurs. 
Farmer perception of the main constraints and cultural practices in sweet potato production in Burkina Faso

\section{ABSTRACT}

Objective: The production of the sweet potato crop, Ipomoea batatas (L.) Lam. is expanding rapidly but still faces many biotic constraints. To better cope with these constraints, it is critical to precisely know and prioritize these constrains

Methodology and results: a survey was carried out between July 2012 and October 2016, with 200 producers in the five main provinces producing sweet potato in Burkina Faso (Gourma, Kénédougou, Kouritenga, Nahouri, Sissili). The results indicate that for producers, insects are the major biotic constraint with varying perceptions from one province to another. Thus, the weevil Cylas puncticollis is seen as the first leaves pest in Nahouri (52. $94 \%)$, Kouritenga and Gourma (75.51\%) while in Sissili and Kénédougou, white flies Bemisia tabaci occupies this position. However, the weevil is seen as the most important pest of tubers in all the provinces. It is also considered by at least $50 \%$ of respondents as the main pest of stems. Cultural practices vary are characterized by a strong application of organic manure, NPK and urea depending on the areas. Crop rotation and the use of synthetic pesticides are cited as methods of controlling the main enemies of sweet potato.

Conclusion and application of findings: This study shows that the producers have the good knowledge of the biotic constraints related to the sweet potato production. This is likely to facilitate the development of participatory and effective strategies of sustainable management of the weevil in Burkina Faso. The results will feed the producer support networks and serve to better define the national strategy for developing the sector. Keywords: Burkina Faso, sweet potato, production constraints, insect pests, Cylas puncticollis, producer's perception.

\section{INTRODUCTION}

Au Burkina Faso, la patate douce est la principale plante à tubercule produite devançant l'igname et la pomme de terre (DGESS, 2014). La production se fait presque dans toutes les zones du pays mais les plus grandes zones de production se trouvent dans les provinces de la Sissili et du Nahouri, dans le sud du pays, et dans la province du Kénédougou au sudouest (DGESS, 2014). La patate douce constitue une importante source de revenus pour les femmes notamment du fait de leur forte implication dans les activités de post-récolte (commercialisation et transformation). La majorité des variétés produites sont à chair blanche, mais des variétés de patate douce à chair orange sont de plus en plus proposées aux producteurs. Du fait de leur richesse en $\beta$ caroténoïde, un précurseur de la vitamine $A$ ( Low et al., 2007 ; Burri, 2011), les variétés à chair orange suscitent beaucoup d'intérêt. Elles constituent un moyen de réduction de la carence en vitamine $A$, qui au Burkina Faso, affecte au moins $30 \%$ des enfants de moins de 5 ans (INSD, 2013). La production de la patate douce au Burkina Faso est passée de 50000 t/an dans les années 90 à 160000 t en 2013
(DGESS, 2014), mais cet accroissement semble dû pour l'essentiel à l'augmentation des surfaces emblavées. En effet les rendements au plan national sont en moyenne de $15 t /$ ha contre $25 t /$ ha pour les variétés améliorées testées en station (Djinet et al., 2015). Le faible rendement des variétés de patate douce en milieu paysan s'explique principalement par la pauvreté des sols, l'insuffisance d'eau, les maladies (virales et fongiques) et les insectes ravageurs (Alghali et al., 1994, Okonya et al., 2014). Les charançons du genre Cylas constituent le principal ravageur de la patate douce dans le monde (Jansson et 1987, Chaltant et al 1990, Fuglie, 2007). En Afrique, Cylas puncticollis Boheman et Cylas brunneus Fabricius sont les principaux ravageurs connus (Kiiza et al., 2009, Okonya et al., 2016). En plus des charançons du genre Cylas, les chenilles de certains lépidoptères tels qu'Acraea acerata Hew. (Lepidoptera : Nymphalidae) et Agrius convolvuli Linnaeus (Lepidoptera : Sphingidae) peuvent causer d'importants dégâts sur cette culture (Stathers et al., 2013 ; Okonya et al., 2014). Parmi les vecteurs de maladies virales, Bemisia tabaci Gennadius 
(Hemiptera : Aleyrodidae) est le plus redouté (Fuglie, 2007 ; Gamarra et al., 2016). Hormis les insectes, d'autres arthropodes tels que les iules et certains mammifères (rongeurs, singes...) sont souvent cités par les producteurs comme étant des ravageurs de la patate douce (Nsibande et al., 1999, Okonya et al., 2014). Au Burkina Faso, on ne dispose jusqu'ici d'aucune liste exhaustive des contraintes biotiques de la patate douce. Les seules informations proviennent des témoignages de certains

\section{MATERIEL ET METHODES}

Sites de l'étude : Les enquêtes ont été réalisées dans cinq régions administratives du Burkina Faso contribuant à plus de $90 \%$ de la production nationale de patate douce producteurs. La connaissance des contraintes de production d'une culture est cependant une étape préliminaire importante pour la mise au point de stratégies efficaces de protection. La présente étude, menée sous forme d'enquêtes, a permis de recueillir les perceptions que les producteurs de patate douce ont des contraintes rencontrées sur cette culture dans les principales zones de production au Burkina Faso.

(DGESS, 2014). Les régions, les provinces, les villages retenus ainsi que les productions annuelles de patate douce sont résumés dans le tableau 1.

Tableau 1 : Liste des villages enquêtés par régions et provinces et productions annuelles de patate douce des régions (DGESS, 2014)

\begin{tabular}{|l|l|l|l|}
\hline $\begin{array}{l}\text { Régions } \\
\text { d'enquêtes }\end{array}$ & Provinces & Villages retenus & $\begin{array}{l}\text { Productions de patate douce (tonne) } \\
\text { dans la région en 2014 }\end{array}$ \\
\hline Centre Sud & Nahouri & Tiébélé & 1764 \\
\hline Centre Ouest & Sissili & $\begin{array}{l}\text { Yelbouga, Yoro, Boura, } \\
\text { Benaverou, Mouna. }\end{array}$ & 16570 \\
\hline Centre Est & Kouritenga & Nakaba, Daltenga & 3153 \\
\hline Est & Gourma & Diabo & 2874 \\
\hline Hauts Bassins & Kénédougou & $\begin{array}{l}\text { Sokouraba, Samôgôyiri, } \\
\text { Mahon, Kôlôkô, Samôgôgouan } \\
\text { Sikorla-diegandougou. }\end{array}$ & 100700 \\
\hline
\end{tabular}

Méthodologie: L'étude a été réalisée sous forme d'enquête à l'aide de questionnaire auprès des producteurs de patate douce. Le questionnaire était partiellement ouvert car les enquêtés avaient la liberté de donner d'autres types d'information sur la production de la patate douce. Dans chacune des provinces de la Sissili, du Nahouri et du Kénédougou qui sont de grandes zones de production, 50 producteurs ont été enquêtés. Par contre dans les provinces du Kouritenga et du Gourma où la production est plus faible, 50 producteurs ont été interviewés dans ces deux provinces. La proximité de la commune de Diabo dans le Gourma avec la province du Kouritenga $(30 \mathrm{~km})$ a motivé le regroupement des données de ces deux provinces. Également, la production de la patate douce dans les provinces du Gourma et du Kouritenga était inferieure à celles des trois autres provinces avant la campagne agricole 2013- 2014 (MASA, 2013). La recherche bibliographique a permis de répertorier les principales contraintes de la patate douce. Les images de ces ravageurs imprimées en couleurs, étaient présentées aux producteurs pour faciliter leur reconnaissance. Le critère de choix des villages s'est fait suivant l'importance de la production de la patate douce tandis que celui des producteurs était basé sur l'expérience d'au moins trois années dans la production. Les agents de vulgarisation du Ministère de l'Agriculture ont été sollicités pour la sélection des villages et des producteurs suivant les critères suscités. La méthode de collecte des données était l'interview individuelle. Elle a consisté dans un premier temps à noter l'identité et les caractéristiques de chaque enquêté (nom, âge, sexe, niveau d'instruction) et dans un second temps, les informations en rapport avec la production. L'interview s'est fait en Français et en Moore dans le Gourma, le Kouritenga, le Nahouri et la Sissili tandis que dans le Kénédougou le Français et le Dioula ont été utilisés. Au cours des entretiens, il a été demandé aux producteurs de classer par ordre d'importance les principaux ravageurs des différents organes de la patate douce. La méthode d'Okonya et al (2014) a été utilisée pour classer les ravageurs des feuilles, des tiges et des 
tubercules de la patate douce. Ainsi les producteurs donnaient un rang à chaque ravageur en fonction de son importance :

1er: Un ravageur très important

$2^{\text {ème }}:$ Un ravageur moyennement important

Зème : Un ravageur peu important

4ème: Un ravageur qui n'est pas important

Ensuite les producteurs ont été interrogés sur les techniques culturales qu'ils adoptent dans la production de la patate douce. Parmi ces techniques culturales, il y a l'utilisation de la fumure organique, des engrais minéraux, la rotation des cultures, la monoculture. Ils ont été interrogés aussi sur l'utilisation des insecticides, la présence des variétés résistantes aux charançons et la conservation des tubercules.

\section{RESULTATS}

Caractéristiques des producteurs enquêtés: Le tableau 2 résume les caractéristiques générales des producteurs enquêtés. II y a plus d'hommes que de femmes qui cultivent la patate douce dans toutes les
Traitement des données : Le tableur Excel 2013 a été utilisé pour saisir les données et pour calculer les moyennes et les pourcentages. Pour chaque ravageur le pourcentage est calculé en fonction du nombre de producteurs ayant attribué le même rang à ce ravageur. Ainsi, la formule $100 \times n i / n t$ où ni est le nombre de producteurs qui ont choisi le rang et nt le nombre total des producteurs est utilisée (Ebregt, 2004a). Une analyse descriptive des moyennes est faite à l'aide $\mathrm{du}$ logiciel SAS version 9.1 (SAS 2003) pour voir s'il y a une corrélation entre les rangs des ravageurs dans les différentes localités. La valeur de $\mathrm{R}^{2}$ a servi à apprécier ces corrélations.

Tableau 2: Caractéristiques générales des producteurs enquêtés

\begin{tabular}{|c|c|c|c|c|}
\hline Variables (\%) & Nahouri & Sissili & Kouritenga-Gourma & Kénédougou \\
\hline \multicolumn{5}{|l|}{ Sexe } \\
\hline $\begin{array}{l}\text { Masculin } \\
\text { Féminin }\end{array}$ & $\begin{array}{l}94,11 \\
5,88\end{array}$ & $\begin{array}{l}88 \\
12\end{array}$ & $\begin{array}{l}100 \\
0\end{array}$ & $\begin{array}{l}100 \\
0\end{array}$ \\
\hline \multicolumn{5}{|l|}{ Age } \\
\hline $\begin{array}{l}15-30 \\
30-40 \\
40-50 \\
50 \text { et plus }\end{array}$ & $\begin{array}{l}15,68 \\
41,17 \\
31,37 \\
11,76 \\
\end{array}$ & $\begin{array}{l}34 \\
32 \\
18 \\
16\end{array}$ & $\begin{array}{l}22,44 \\
30,61 \\
30,61 \\
16,32 \\
\end{array}$ & $\begin{array}{l}28 \\
32 \\
30 \\
10 \\
\end{array}$ \\
\hline \multicolumn{5}{|c|}{ Niveau d'instruction } \\
\hline $\begin{array}{l}\text { Primaire } \\
\text { Secondaire } \\
\text { Alphabétisé } \\
\text { Non Alphabétisé }\end{array}$ & $\begin{array}{l}19,60 \\
13,72 \\
3,92 \\
62,74 \\
\end{array}$ & $\begin{array}{l}26 \\
6 \\
4 \\
64 \\
\end{array}$ & $\begin{array}{l}42,85 \\
16,32 \\
0 \\
40,81 \\
\end{array}$ & $\begin{array}{l}22 \\
12 \\
10 \\
56 \\
\end{array}$ \\
\hline
\end{tabular}

provinces enquêtées. La tranche d'âge la plus active dans la production de la patate douce est comprise entre 30 et 50 ans. La majorité des enquêtés ne sont pas alphabétisés même dans les langues locales.
Principaux ravageurs des feuilles de la patate douce : Les principaux ravageurs des feuilles de la patate douce énumérés par les producteurs dans les différentes localités sont les insectes incluant les charançons, les aphides, les mouches blanches, les chenilles défoliantes et les sautériaux. Le charançon $C$. puncticollis occupe le premier rang des ravageurs des feuilles dans le Nahouri $(52,94 \%)$, dans le Kouritenga et dans le Gourma $(75,51 \%)$. En revanche, dans la Sissili et le Kénédougou, la mouche blanche $B$. tabaciest classée au premier rang des ravageurs de cette partie de la plante respectivement par 70 et $54 \%$ des producteurs (Figure1). A la suite des charançons et des mouches blanches viennent les chenilles défoliatrices et les aphides. Cependant, l'importance des ravageurs des feuilles varie d'une localité à une autre, sauf dans la Sissili et dans le Kénédougou où les ravageurs semblent avoir la même importance (Tableau 3). 
Koussoube et al, J. Appl. Biosci. 2018 Perception paysanne des principales contraintes et pratiques culturales en production de patate douce au Burkina Faso.

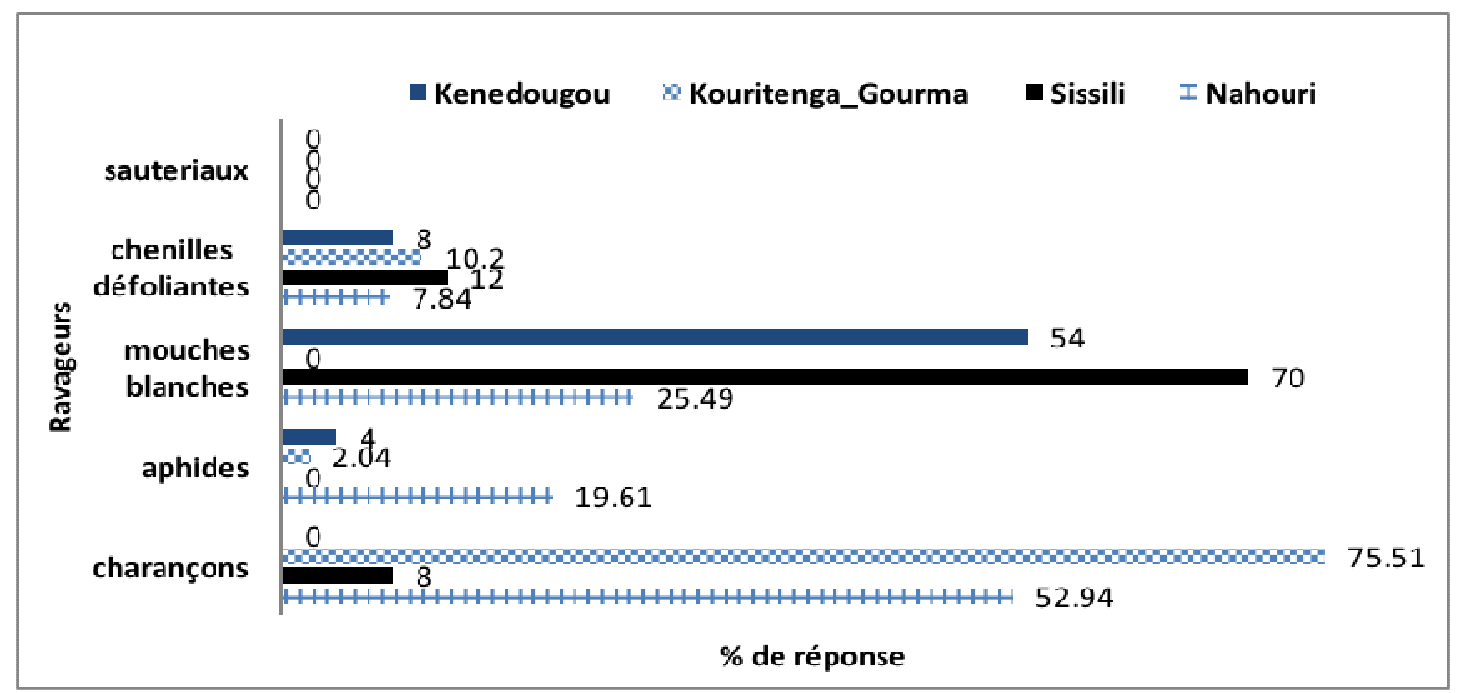

Figure 1 : Importance relative des insectes ravageurs des feuilles de la patate douce telle que perçue par les producteurs de diverses localités du Burkina Faso

Tableau 3 : Corrélation entre les réponses des producteurs de différentes localités sur les principaux ravageurs des feuilles

\begin{tabular}{l|l|l|l|l}
\hline & Nahouri & Sissili & Kouritenga_Gouma & Kénédougou \\
\hline Nahouri & 1 & & & \\
Sissili & $0,16^{* *}$ & 1 & & \\
Kouritenga_Gourma & $0,84^{* * *}$ & $-0,22$ & 1 & 1 \\
Kénédougou & $0,84^{* * * *}$ & $0,98^{* * *}$ & $-0,35$ & 1 \\
\hline
\end{tabular}

${ }^{* * *}=$ forte corrélation $;{ }^{* *}=$ corrélation moyenne $;{ }^{*}=$ faible corrélation

Principaux ravageurs des tiges de la patate douce : II y a une forte corrélation entre les réponses des producteurs de toutes les zones enquêtées sur les principaux ravageurs des tiges (Tableau 4). Le charançon, C. puncticollis est classé au premier rang selon les producteurs interviewés $(98,04 \%$ dans le Nahouri, $100 \%$ dans la Sissili, $67,35 \%$ dans le Kouritenga et le Gourma et $80 \%$ dans le Kénédougou). A la suite des charançons viennent les chenilles foreuses des tiges (Figure 2).

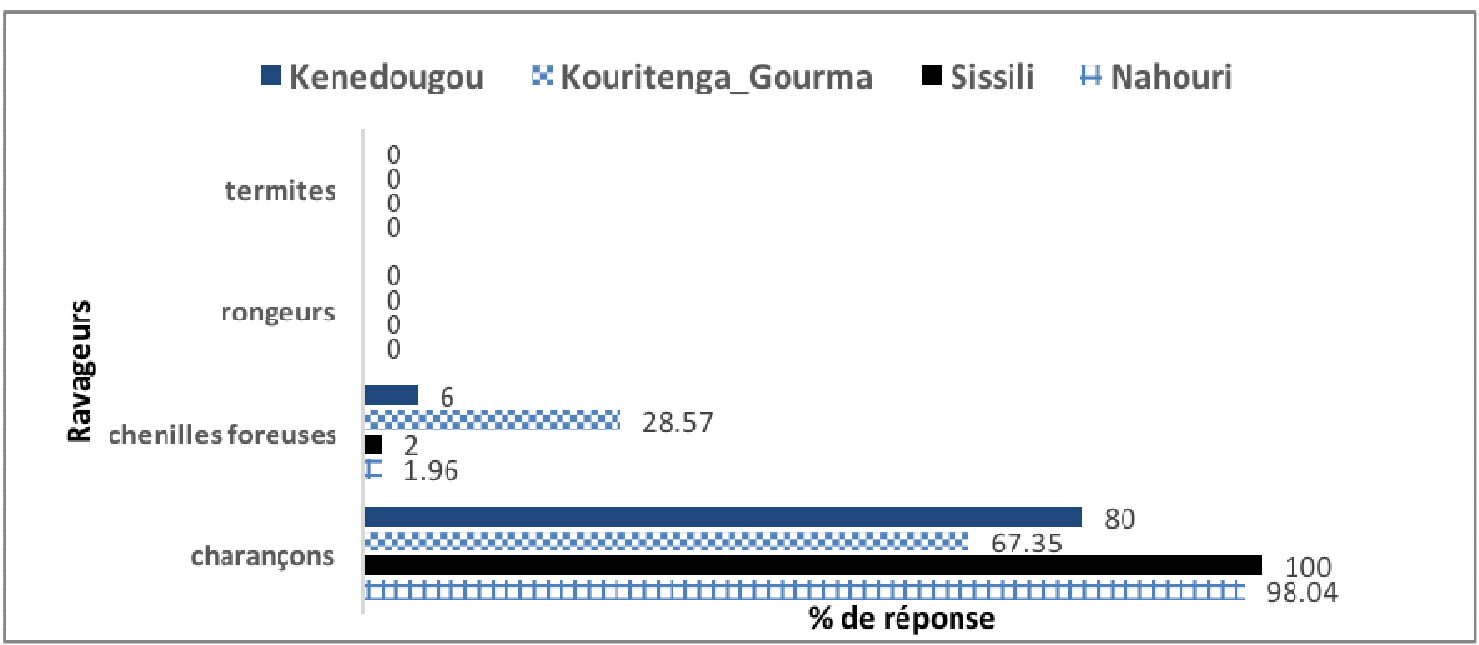

Figure 2 : Importance relative des insectes ravageurs des tiges de la patate douce telle que perçue par les producteurs de diverses localités du Burkina Faso 
Koussoube et al, J. Appl. Biosci. 2018 Perception paysanne des principales contraintes et pratiques culturales en production de patate douce au Burkina Faso.

Tableau 4 : Corrélation entre les réponses des producteurs de différentes localités sur les principaux ravageurs des tiges

\begin{tabular}{l|l|l|l|l}
\hline & Nahouri & Sissili & Kouritenga_Gouma & Kénédougou \\
\hline Nahouri & 1 & & & \\
Sissili & $1^{*+*}$ & 1 & & \\
Kouritenga_Gouma & $0,91^{*+*}$ & $0,91^{*+*}$ & 1 & 1 \\
Kénédougou & $0,99^{*+*}$ & $0,99^{*+*}$ & $0,93^{*+*}$ & 1 \\
\hline
\end{tabular}

${ }^{* * *}=$ forte corrélation $;{ }^{* *}=$ corrélation moyenne $;{ }^{*}=$ faible corrélation

Principaux ravageurs des tubercules de la patate douce : Une forte corrélation existe entre les réponses des producteurs sur les principaux ravageurs des tubercules dans toutes les zones d'enquêtes (Tableau 5). En effet, dans toutes les localités, le charançon $C$. puncticollis est cité comme étant le principal ravageur des tubercules. Les autres ravageurs d'importance secondaire cités de façon très variable selon les localités incluent les termites et les mille-pattes ou iules (Figure 3).

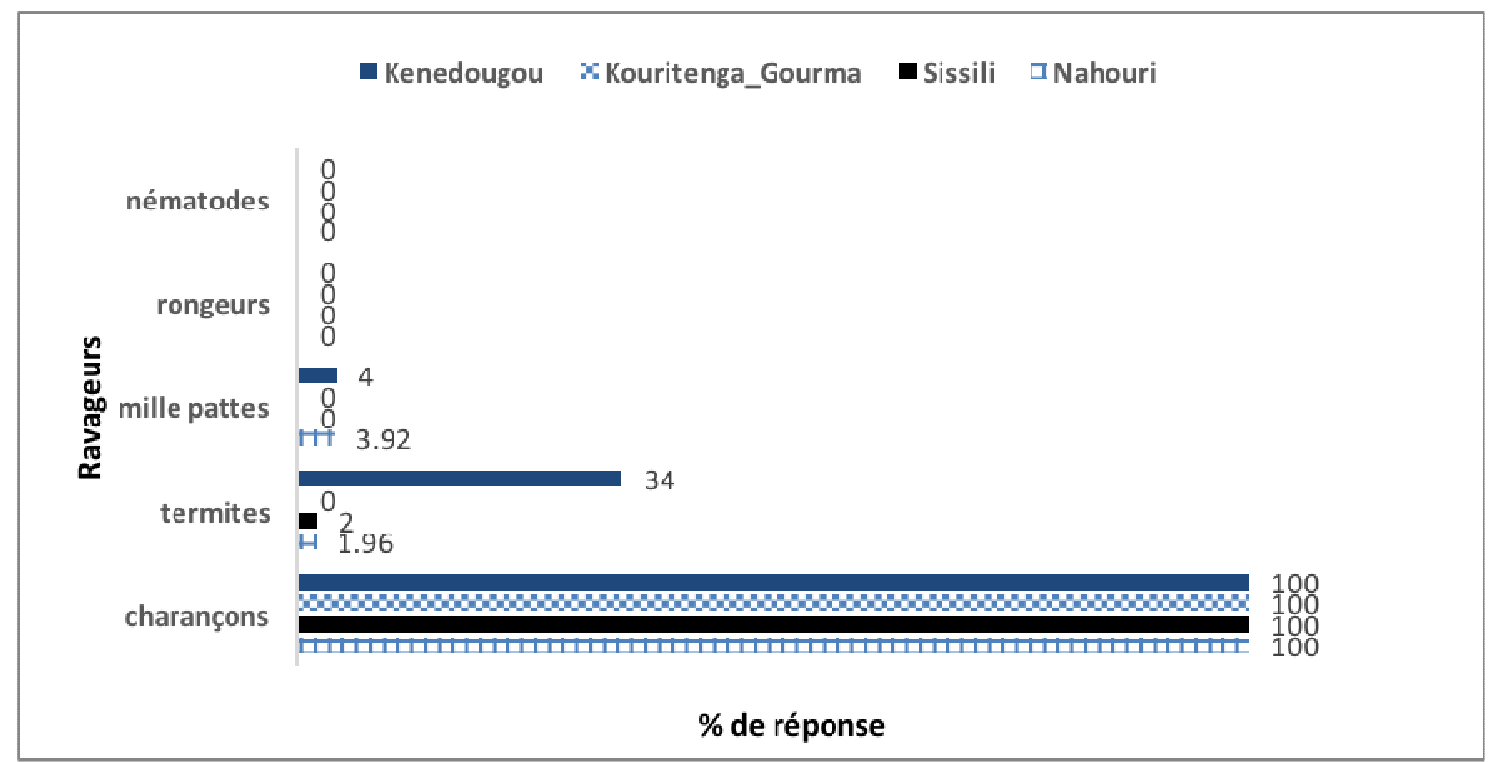

Figure 3: Importance relative des insectes ravageurs des tubercules de la patate douce telle que perçue par les producteurs de diverses localités du Burkina Faso

Tableau 5 : Corrélation entre les réponses des producteurs de différentes localités sur les principaux ravageurs des tubercules

\begin{tabular}{l|l|l|l|l}
\hline & Nahouri & Sissili & Kouritenga_Gouma & Kénédougou \\
\hline Nahouri & 1 & & & \\
Sissili & $0,99^{*+*}$ & 1 & & \\
Kouritenga_Gouma & $0,99^{*+*}$ & $0,99^{* * *+}$ & 1 & 1 \\
Kénédougou & $0,94^{* * *}$ & $0,94^{*+*}$ & $0,94^{*+*}$ & 1 \\
\hline
\end{tabular}

${ }^{* * *}=$ forte corrélation $;{ }^{* *}=$ corrélation moyenne $;{ }^{*}=$ faible corrélation

Pratiques culturales en production de patate douce dans les zones d'enquête : Les paysans utilisent des engrais organiques et minéraux dans la culture de la patate dont les quantités et types varient en fonction des localités (tableau 6). Ainsi la fumure organique et le NPK sont peu utilisés dans la province du Kénédougou comparativement aux autres localités (respectivement 54 et $56 \%$ des enquêtés). L'urée par contre est très utilisée dans la province du Nahouri et très peu dans celle de la Sissili (Tableau 6). La majorité des paysans cultivent la patate douce en monoculture (tableau 6). Les producteurs de patate douce font recours à divers moyens de protection contre les insectes ravageurs. En premier ressort, la lutte chimique avec des insecticides de 

en production de patate douce au Burkina Faso.

synthèse est utilisée par $75-97 \%$ des personnes interviewées (Tableau 6). Pour les enquêtés, il n'y a pas de variétés de patate douce non attaquées par les charançons. Toutefois, ils estiment que leurs variétés locales sont résistantes aux charançons. Les producteurs mentionnent également la rotation des cultures comme un moyen de lutte contre les charançons, qui en fonction des zones, serait pratiquée entre la patate douce le mil, le sorgho, le maïs, le riz, les cultures maraîchères (tomate, piment, gombo, l'oignon...), l'arachide et le niébé. La rotation est perçue comme un moyen de contrôle des charançons mais ils affirment aussi qu'elle permet d'améliorer d'une manière générale les rendements des tubercules. L'analyse des pratiques post récolte montre que les tubercules de patate douce ne sont pas conservés par la majorité des producteurs. La quantité de tubercules qu'ils conservent serait très faible et la durée de conservation ne dépasserait pas trois mois. Les méthodes de conservation incluent l'utilisation de fosses et la conservation à l'air libre dans les concessions. La conservation dans les champs n'est pas pratiquée à cause de la sécheresse qui survient souvent à la fin de la saison hivernale ce qui augmente les dégâts des charançons (Tableau 6).

Tableau 6 : Répartition des pratiques culturales en production de patate douce dans les localités d'enquête

\begin{tabular}{l|l|l|l|l}
\hline \multirow{2}{*}{ Site } & \multicolumn{4}{|l}{ Pourcentage (\%) des réponses/province } \\
\cline { 2 - 5 } & Nahouri & Sissili & Kouritenga-Gourma & Kénédougou \\
\hline Fumure organique & 92,16 & 68 & 100,00 & 54 \\
NPK & 70,59 & 94 & 93,88 & 56 \\
Urée & 96,08 & 20 & 44,90 & 56 \\
Monoculture & 94,12 & 80 & 69,39 & 90 \\
Insecticides de synthèse & 96,08 & 84 & 75,51 & 82 \\
Variétés résistantes aux charançons & 19,61 & 4 & 2,04 & 16 \\
Rotation des cultures & 94,12 & 94 & 61,22 & 88 \\
Conservation des tubercules & 27,45 & 8 & 28,57 & 44 \\
\hline
\end{tabular}

\section{DISCUSSION}

Cette étude a mis en évidence les contraintes biotiques de production de la patate douce telles que perçues par les producteurs des principales zones de production au Burkina Faso. Même si les producteurs semblent connaître les principales espèces d'insectes s'attaquant à la patate douce, ils ont souvent des difficultés à identifier les organes des plantes qui sont vulnérables à chaque ravageur. Nos résultats ont montré que la perception que les producteurs ont des ravageurs de la patate douce varie d'une zone à une autre. Dans tous les cas, deux espèces de ravageurs majeures, la mouche blanche, $B$. tabaci, et le charançon, C. puncticollis ont été identifiées par les producteurs des zones d'enquête. Les producteurs du Nahouri, du Kouritenga et du Gourma ne considèrent pas la mouche blanche, comme un ravageur important des feuilles de la patate douce contrairement à ceux de la Sissili et du Kénédougou. Cette différence de perception pourrait s'expliquer par plusieurs raisons. D'une part, elle pourrait être liée à une importance variable de l'insecte d'une zone à une autre et d'autre part, elle pourrait aussi provenir d'une méconnaissance de la biologie des mouches blanches par une grande partie des producteurs des localités concernées. En accord avec nos résultats, des études réalisées en
Ouganda ont montré que les producteurs de deux localités sur six considéraient Bemisia tabaci comme étant un ravageur d'importance secondaire de la patate douce (Okonya et al., 2014). La méconnaissance de la biologie des insectes ravageurs est un handicap pour les producteurs dans la protection de leur culture. De la même manière, plus de $52,94 \%$ des enquêtés dans la province de la Sissili et $75,51 \%$ dans la province du Kouritenga et du Gourma classent aussi les charançons au 1 er rang des ravageurs des feuilles de la patate douce. Les producteurs remarquent plus facilement les dégâts causés par les larves des charançons sur les tiges et les tubercules puisque les larves sont présentes dans ces organes (Nsibande ,1999 Stathers et al., 2013). Cela amène certains producteurs à lier tous les autres dégâts observés dont ils ne connaissent pas les origines aux larves des charançons. Dans tous les cas, les résultats de notre étude confirment que les dégâts des charançons du genre Cylas sont la principale contrainte de la production de la patate douce dans toutes les zones de production au Burkina Faso et dans le monde (Jansson et 1987, Chaltant et al., 1990, Fuglie, 2007). D'autres ravageurs tels que les iules et les termites sont classés au deuxième rang des ravageurs des tubercules. Les 
termites sont plus actifs dans des sols où la matière organique n'est pas complètement décomposée. Ils peuvent causer d'énormes dégâts aussi sur les tiges et réduire considérablement la reprise des boutures. Les iules profitent des trous issus des émergences des adultes des charançons pour pénétrer dans les tubercules. Ils sont attirés par les tubercules endommagés (Ebregt et al., 2004b). Les iules sont très importants dans la province du Kénédougou probablement en raison d'une pluviométrie plus importante dans cette localité. Ce constat corrobore les travaux de Ebregt et al. (2005) qui ont montré que les iules sont une contrainte majeure de la production de la patate douce au cours de la première saison des pluies en Ouganda où l'humidité est plus stable. En plus de la pluviométrie l'abondance des iules peut dépendre des caractéristiques abiotiques du sol telles que la texture, la matière organique, la teneur en calcium (Demange, 1975, Smit, 1997, Ebregt et al.,2004b). En ce qui concerne les itinéraires culturaux, la monoculture semble être la pratique dominante même si elle est couplée à une

\section{CONCLUSION}

II ressort de cette étude que le charançon C. puncticollis représente la principale contrainte biotique de la production de la patate douce au Burkina Faso. Les autres insectes ravageurs de la patate douce sont les mouches blanches, les chenilles défoliantes, les chenilles foreuses et les termites. Les iules peuvent être aussi des contraintes pour la production de cette culture dans les zones les plus humides. Les résultats de cette étude seront aussi utiles pour les décideurs politiques dans la stratégie nationale de la filière pour une autosuffisance nutritionnelle et alimentaire. Les entreprises et les projets de développement intervenant dans la filière patate douce

\section{REMERCIEMENTS}

Nous remercions Monsieur Jérôme BELEM Agronome à la retraite et Dr Ali GARANE pour leur collaboration. Nous tenons à remercier aussi les techniciens Théodore OUEDRAOGO et Simon TARPIDGA ainsi que les

\section{BIBLIOGRAPHIE}

Alghali A. M. et Bockarie A., 1994.Cultural practices and perceptions in sweet potato farming and their implications for pest management in southern Sierra Leone. Int. J.Pest Manage. 40, 27 - 30

Burri BJ. 2011. Evaluating sweet potato as an intervention food to prevent vitamin A deficiency. Compr Rev Food Sci Food Saf 10:118 - 130. rotation des cultures par la majorité des répondants. La forte utilisation d'insecticides de synthèse dans toutes les zones d'enquêtes est certainement en lien avec l'importance des contraintes dues aux insectes et surtout celles provenant des attaques du charançon. La proportion de producteurs qui utilise les engrais organiques et inorganiques est élevée dans les zones où les sols sont pauvres. Dans certaines localités comme la province du Nahouri, $96,08 \%$ des enquêtés utilisent l'urée comme fertilisant. Par contre de l'avis de certains auteurs, la patate douce se développe bien sur des sols pauvres et ne nécessite pas un apport important d'engrais organique et/ou inorganique (Gibbon et al., 1985 ; Smit et Matengo, 1995 ; Stathers et al., 2013). L'intérêt réel de telles pratiques mérite d'être déterminé avec précision afin d'optimiser les pratiques en production de patate douce au Burkina Faso. Cela d'autant plus que l'utilisation de certains types de fertilisants pourrait réduire le délai de conservation post récolte des tubercules (Stathers et al, 2013).

pourront utiliser ces résultats pour renforcer leurs dispositifs de formation des agents de vulgarisation et d'appui aux producteurs pour une production plus rentable. En termes de perspectives pour améliorer la production de la patate douce au Burkina Faso, il faudra nécessairement connaitre la bio écologie du charançon ainsi que son impact réel en production et en conservation post récolte de la patate. Ces informations permettront de mieux définir les stratégies d'une gestion efficace et durable du ravageur au Burkina Faso et en Afrique de l'Ouest.

doctorants Apolline SANOU et Adama KABORE du Laboratoire Central d'Entomologie Agricole de Kamboinsé pour leurs contributions lors de la réalisation de cette activité.

Chaltant R.B., Jansson R.K., Seal D.R et Schalk J.M., 1990. Ecology and main agement to sweet potato insect. Annu. Rev.Entomol.35, 157-189.

Demange, J.M., 1975, Les Myriapodes Diplopodes nuisibles à l'arachide au Sénégal. O/eagineux 30:19-2 4. 
DGESS, 2014 Résultats définitifs de la campagne agricole et de la situation alimentaire et nutritionnelle en 2013/2014. MASA.77p

Djinet A.I., NANA R., TAMINI Z., BADIEL B., 2015. Étude comparée des paramètres agromorphologiques de dix (10) variétés de patate douce (Ipomea batatas (L) Lam cultivées au champ dans deux (2) conditions climatiques au Tchad et au Burkina Faso. Int.J. Biol.Chem. Sci.9(3) : 12431251.

Ebregt, E., P.C. Struik, P.E. Abidin et B. Odongo, 2004a. Farmers' information on sweet potato production and millipede infestation in north-eastern Uganda. I. Associations between spatial and temporal crop diversity and the level of pest infestation. NJAS Wageningen Journal of Life Sciences 52: 47-68.

Ebregt, E., Struik P.c., Abidin P.E et Odongo B., 2004b. Farmers' information on sweet potato production and millipede infestation in north-eastern Uganda. II. Pest incidence and indigenous control strategies. NJAS - Wageningen Journal of Life Sciences 52: 69-84.

Ebregt, E., Struik, P., Odongo, B. et Abidin, P., 2005. Pest damage in sweet potato, groundnut and maize in north-eastern Uganda with special reference to damage by millipedes (Diplopoda). NJAS - Wageningen Journal of Life Sciences, 53(1), pp. 49-69.

Fuglie, K.O. 2007. Priorities for sweet potato research in developing Countries: Results of a survey. HortScience 42:1200-1206.

Gamarra, H., Carhuapoma, P., Kreuze, J., Kroschel, J., 2016. Pest distribution and risk atlas for Africa. Potential global and regional distribution and abundance of agricultural and horticultural pests and associated biocontrol agents under current and future climates. Lima (Peru). International Potato Center (CIP). ISBN 978-92-9060-476-1 DOI 10.4160/9789290604761-8. pp. 100-113

Gibbon D. et Pain A., 1985. Crops of the Drier Regions of the Tropics. Longman, London, $157 \mathrm{pp}$.

INSD, 2013. Annuaire statistique 2011. Ministère de l'Économie et des finances. 420p

Jansson R.K., Brvan H.N., Sorensen K.A., 1987. Within vine distribution and age of the sweet potato to weevil Cylas formicarius eiegantulus
(Coleoptera: Curcutionidae) on four cultivars of sweet potato in southern Florida.

Kiiza, B., Mwanga, R.O.M., Kisembo, L., Kreuze, J., Labarta, R. and Ghislain, M. 2009. Analysis of economic implications of biotech sweet potato in the Great Lakes Region to control weevil and virus disease damage. Uganda Country Report

Low J.W., Arimond M., Osman N., Cunguara B., Zano F., Tschirley D., 2007. A food-based approach introducing orange-fleshed sweet potatoes increased vitamin $\mathrm{A}$ intake and serum retinol concentrations in young children in rural Mozambique. J Nutr 137:1320- 1327.

MASA., 2013. Résultats définitifs de la campagne agricole et de la situation alimentaire et nutritionnelle 2012/2013. 49p.

Nsibande M.L., 1999. Sweet potato, Ipomoea batatas (L), cropping practices and perceived production constraints in Swaziland: Implications for pest management, International Journal of Pest Management, 45:1, 29-33.

Okonya J.S., Robert O.M., Mwanga R. O.M., Syndikus K., Kroschel J., 2014. Insect pests of sweet potato in Uganda: farmers 'perceptions of their importance and control practices. SpringerPlus 2014 3:303.

Smit N.E.J.N et Matengo L.O., 1995. Farmers cultural practices and their effect pest control in sweet potato in south Nyanza, Kenya. Int. J. Pest Manage. 41, 2-7.

Smit, N.E.J.M. 1997. Integrated Pest Management for sweet potato in Eastern Africa PhD Thesis, Wageningen University, The Netherlands.

Stathers T., Low., J., Mwanga, R., Carey, T., David., S., Gibson, R., Namanda, S., McEwan, M., Bechoff., A., Malinga, J., Benjamin, M., Katcher, H., Blakenship, J., Andrade, M., Agili, S., Njoku, J., Sindi, K., Mulongo, G., Tumwegamire, S., Njoku, A., Abidin, E., Mbabu, A. 2013.Tout ce que vous avez toujours voulu savoir à propos de la patate douce : Atteindre les agents du changement, manuel de formation des formateurs (FdF). Centre International de la Pomme de Terre, Nairobi, Kenya. 7 vols. xviii, $454 \mathrm{p}$. 